\title{
P4: Neue Wege in der praktischen Gesundheits- und Krankenpflege und Gesundheits- und Kinderkrankenpflegeausbildung - Der HELIOS Kompetenzkompass
}

\author{
Mandy Dietel · Bettina Duri · Waltraud Georg • \\ Birgit Göschel • Grit Höser • Manja Riese • \\ Cornelia Wossidlo
}

Online publiziert: 23. Oktober 2013

(C) Springer-Verlag Wien 2013

Einleitung: Im Rahmen der internationalen Kompetenzdebatte in der beruflichen Bildung wurde 2009 ein neues Instrument in den HELIOS Kliniken entwickelt, um die Kompetenzentwicklung der Schüler in der Gesundheitsund (Kinder-)Krankenpflege zu erfassen und darzustellen. Methodik: Die Grundlage des Kompetenzkompasses bildetet die, auf der Ausbildungs- und Prüfungsverordnung basierenden, zwölf Themenbereiche der theoretischen Ausbildung und die 4 Kompetenzdimensionen nach Krankenpflegegesetz.

Vor jeder praktischen Ausbildungssequenz legen die Schüler zusammen mit dem Praxisanleiter in einem Einführungsgespräch Lernziele fest, die in einem Zwischen- und Abschlussgespräch überprüft, reflektiert und protokolliert werden.

In der Skills-Übersicht werden klinisch-praktische Fertigkeiten dokumentiert, die der Schüler unter Anleitung wiederholt trainiert.

Die Einschätzung der Kompetenzausprägung eines Schülers wird am Ende der Ausbildungssequenz vom Pra- xisanleiter auf einem themenbereichsbezogenen Kompetenzstrahl dokumentiert. Der Schüler überträgt diese Einschätzung in Eigenverantwortung in eine Grafik, sodass sich eine Entwicklungskurve ergibt.

Ergebnisse und Diskussion: Die vorrangig tätigkeitsorientierte Ausbildung in der Pflege soll mit der Einführung des Kompetenzkompasses abgelöst werden, da sich das komplexe berufliche Handeln in der Pflege durch die Fach-, Selbst-, Methoden- und Sozialkompetenz widerspiegelt. Es wird eine objektive Bewertung und die Sicherung einer hohen Qualität durch die kontinuierliche Begleitung und transparente Dokumentation der Ausbildung gewährleistet. Der Kompetenzkompass fördert die Eigenverantwortlichkeit des Schülers, visualisiert die persönliche Entwicklung in den drei Ausbildungsjahren und strukturiert die praktische Ausbildung anhand von Lernzielen.

Im Jahr 2011 wurde der Kompetenzkompass in den HELIOS Kliniken implementiert, die erste Evaluation folgte 2012 und 2013 wurden Review-Bögen eingeführt, um praktische Ausbildungssequenzen rückblickend zu betrachten.
M. Dietel $(\bowtie) \cdot$ B. Duri $\cdot$ W. Georg $\cdot$ B. Göschel $\cdot$ G. Höser

M. Riese $\cdot$ C. Wossidlo

AG Kompetenzen, HELIOS Kliniken GmbH, Berlin, Deutschland

E-Mail: mandy.dietel@helios-kliniken.de 